through $(x, y)$ into four equal squares; in one of these at least the average density is greater than $2 \varepsilon$. It follows that at each point of $E_{2}$, either:

$$
\begin{aligned}
& \text { either } \\
& \text { (85) } \lim _{h \rightarrow 0} \frac{\mu_{c}[H G(x, y, 3 \eta) R(x, y ; h, h)]}{h^{2}}>2 \varepsilon \\
& \text { or } \quad \lim _{h \rightarrow 0} \frac{\mu_{e}[H G(x, y, 3 \eta) R(x, y ; h,-h)]}{h^{2}}>2 \varepsilon
\end{aligned}
$$

Hence one at least of $(85)$ and (86) must be true in a set $E_{3}$ of positive outer measure; and we may suppose it is (85), for in the other case we could consider $\Phi(x, y)=-F(x,-y)$. . . .

Let $\left(x_{1}, y_{1}\right)$ be a point of $\boldsymbol{E}_{3}$. There is a sequerice $\eta_{m},\left|l_{n}\right|<1, \delta$, $l_{n} \rightarrow 0$, such that

$$
\frac{\mu_{e}\left[H G\left(x_{1}, y_{1}, 3 \eta\right) R\left(x_{1}, y_{1} ; l_{n}, l_{n}\right)\right]}{l_{n}^{2}}>2 \varepsilon .
$$

Hence for each $n$ there is a point $\left(x_{1}+h_{n}, y_{1}+k_{n}\right)$, say, lying in $H G\left(x_{1}, y_{1}, 3 \eta\right) R\left(x_{1}, y_{1} ; l_{n}, l_{n}\right)$ and such that

$$
h_{n}|>\varepsilon| l_{n}|, \quad| k_{n}|>\varepsilon| l_{n} \mid
$$

It follows that

$$
h_{n} k_{n}>0, \quad \varepsilon\left|h_{n}\right|<\left|k_{n}\right|<\varepsilon^{\prime}\left|h_{n}\right|
$$

and from $(80)$

$$
F\left[R\left(x_{1}, y_{1} ; h_{n}, k_{n}\right)\right]-\underline{D}\left(x_{1}, y_{1}\right) h_{n} k_{n} \mid>3 \eta h_{n} k_{n}
$$

Comparing this with (82) and (83) we have

$$
F\left[R\left(x_{1}, y_{1} ; h_{n}, k_{n}\right)\right]>(A+3) \eta h_{n} k_{n}
$$

We note finally that $\left(x_{1}+h_{n}, y_{1}+k_{n}\right)$ belongs to $H$. From this point the argument is exactly parallel to those used in proving Theorems 1 and 3 ; the detail is left to the reader.

Note. The theorem obtained by replacing ,any square" by ,any" rectangle" in the enunciation of Theorem 4 is certainly false; this may be seen by considering the case of appositive function of rectangles (for example $f f(x ; y) d x d y$ ) which has at a set of positive mear.: sure $\vec{D}(x, y)=+\infty, D(x, y)$ fnite.

\section{Sur les théorèmes de séparation dans la Théorie des ensembles.}

Par

Casimir Ku ratowski (Warszawa).

Soit $\boldsymbol{A}$ une famille' de sous-ensembles d'un espace composé d'éléments arbitraires. On dit que cette famille satisfait au premier thérème de séparation, lorsqu'à chaque couple d'ensembles disjoints $A_{1}$ et $A_{2}$ appartenant à $A$ correspond un ensemble $B$ qui, ainsi que son complémentaire, appartient à $\boldsymbol{A}$ et qui satisfait aux formules $A_{1} \subset B$ et $B A_{2}=0$. Le deuxième théorème de séparation est satisfait, lorsqu'à chaque couple d'ensembles $A_{1}$ et $A_{2}$ appartenant à $\boldsymbol{A}$ correspond un couple d'ensembles disjoints $C_{1}$ et $C_{2}$ dont les complémentaires appartiennent à $A$ et qui remplissent les formules $A_{1}-A_{2} \subset C_{1}$ et $A_{2}-A_{1} \subset C_{2}$.

Des exemples surtout importants de familles satisfaisant aux deux théorèmes de séparation présentent: la famille des ensembles boreliens de classe multiplicative $\beta>0{ }^{1}$ ), celle des ensembles analytiques ${ }^{2}$ ), celle des ensembles projectifs de classe $C P\left(A^{3}\right.$ ).

1) c. à d. $G_{\delta}, F_{\sigma \delta}, G_{\delta \sigma \delta}$ etc. Les théorèmes de séparation pour ces familles d'ensembles ont été démontrés par MM. Lusin et Sierpiński. Voir W. Sierpiński, Fund. Math. 6 (1924), p. 2 et Bulet. Soc. St. de Cluj 6 (1932), p. 461, et N. Lusin, Fund. Math. 16 (1930), pp. 57 et 60.

Il importe de remarquer que la famille des ensembles fermés satisfait au deuxième théorème de séparation et cependant - si l'espace est connexe - elle ne satisfait pas au premier: à chaque couple $E_{1}, E_{2}$ d'ensembles fermés correspond un couple $H_{1}, H_{2}$ d'ensembles ouverts disjoints tel que $E_{1}-E_{2} \subset H_{1}$ et $E_{2}-E_{1} \subset H_{2}$ (cf. par exemple ma Topologie I. p. 99, 2 et 6), tandis que l'espace ne contient aucun vrai gous-ensemble non vide qui soit simultanément fermé et ouvert.

2) Les théorèmes de séparation pour les ensembles analytiques ont été démontrés par M. Lusin sous le nom du "premier et deuxième principes". Ils jouent un role fondamental dans la théorie de ces ensembles.

3) Les théorèmes de séparation pour les ensembles $C P C A$ ont été démontrés par M. N ovik off, Fund. Math. 25 (1935), p. 459. 
Appelons théorème de réduction, relatif à une famille $B$ d'ensembles, la proposition suivante: étant donnés deux ensembles $U_{1}$ et $U_{2}$ appartenant à $\boldsymbol{B}$, il existe deux ensembles disjoints $V_{1}$ et $V_{2}$ appartenant aussi à $B$ et tels que $V_{1} \subset U_{1}, \quad V_{2} \subset U_{2}$ et $V_{1}+V_{2}=$ $=U_{1}+U_{2}$.

On montre facilement que, $B$ itant une famille d'ensembles satisfaisant au théorème de réduction, la famille de leurs complémentaires satisfait aux deux théorèmes de séparation $\left.{ }^{1}\right)$. De plus, les familles: des ensembles boreliens d'une classe additive $\beta>0$, celle des ensembles $C A$ et celle des ensembles $P C A$, satisfont au théorème de réfduction. Elles satisfont, comme nous allons démontrer ${ }^{2}$ ), au théorème de réduction généralisé, $\mathrm{c}$. à d. à l'énoncé suivant: étant donnée une suite infinie d'ensembles $U^{1}, U^{2}, \ldots$ appartenant d la famille $\boldsymbol{B}$, il existe dans cette famille une suite d'ensembles disjoints $V^{1}, V^{2}, \ldots$ tels que

$$
V^{n} \subset U^{n} \text { et } \sum_{n=1}^{\infty} V^{n}=\sum_{n=1}^{\infty} U^{n}
$$

Les différentes généralisations des théorèmes de séparation trouvées récemment dans le domaine des ensembles boreliens et projectifs se déduisent toutes du théorème de réduction généralisé.

\section{Théorème de réduction dans la Théorie générale des ensembles.}

Soit $\kappa_{;}$un nombre cardinal donné. Soit $\Phi$ une famille d'ensembles telle que:

$1^{0}$ si $X \epsilon \Phi$ et $Y_{\epsilon} \Phi$, on a $X-Y_{\epsilon} \Phi$,

$2^{0}$ si $\left\{X_{\xi}\right\}$ est une suite (transfinie) de puissance $\left\langle\boldsymbol{s}_{\text {, }}\right.$ d'ensembles appartenant à $\Phi$, la somme $\sum_{\zeta} X_{\zeta}$ appartient à $\Phi$.

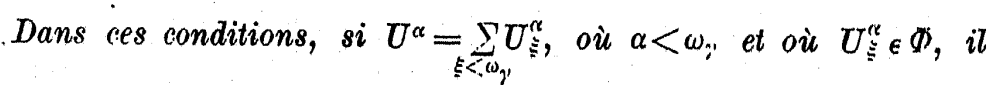
existe une double suite d'ensembles $\nabla_{\xi}^{\Uparrow} \epsilon \Phi$ tels qu'en posant

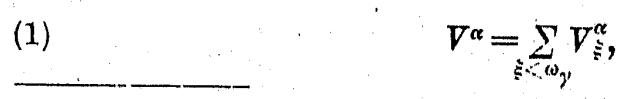

1) Comme l'a remarqué M. Sierpiński, l'implication inverse n'a pas lieu: on ñ'a qu'à considérer comme $B$ la famille de tous les sous-ensembles ouverts de la ligne droite qui ne contiennent pas un point fixe (par ex. le point 0 ).

2) sarts avoir recours d'ailleurs aux théorèmes de séparation, de sorte que la lecture de cette note ne demande pas la connaissance des notes précitées. 
Enfin pour démontrer l'égalité (2), considérons, pour un $x$ donné et appartenant à $\sum_{\alpha} U^{\alpha}$, tous les termes $U_{\xi}^{\alpha}$ qui contiennent $x$. Soit $U_{\xi_{0}}^{\alpha_{0}}$ celui parmi eux dont l'indice $\xi$ est le plus petit. On a donc,

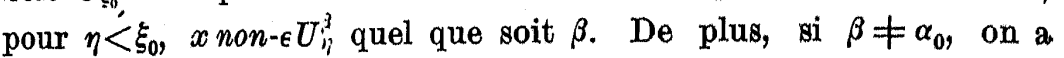

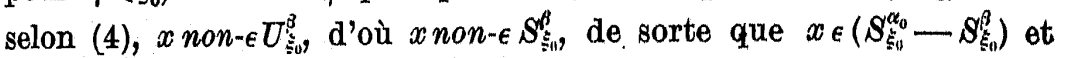
par conséquent $x \in V^{\alpha_{0}}$.

\section{Application aux ensembles boreliens.}

La famille des ensembles boreliens de classe additive $\beta>0$ (dans un espace métrique) satisfait au théoreme de réduotion généralisé.

De plus, si l'espace est séparable de dimension 0, le theoreme est aussi valable pour $\beta=0$ (c. à $\alpha$. pour les ensembles ouverts).

Pour s'en convaincre on n'a qu'à poser dans le théorèrme du No 1: $\gamma=0$ et $\Phi=$ la famille des ensembles ambigus de classe $\beta$ (l'ensemble $V^{n}$ étant défini par la formule (1)).

\section{Application aux ensembles $O A$.}

La famille des ensembles $C A$ satisfait au théorème de réduction généralisé.

Démonstration. Rappelons d'abord que, d'une façon générale, à chaque ensemble analytique $A$ (situé dans un espace complet séparable) correspond une famille d'ensembles fermés $W_{r}$ (le "crible"s), où l'indice $r$ parcourt les nombres rationnels de l'intervalle 01 , telle que $x$ appartient à $A$ dans ce cas et dans ce cas seulement lorsque l'ensemble $M_{x}$ des indices $r$ satisfaisant à la condition $x \in W_{r}$ n'est pas bien ordonné (selon la grandeur des nombres rationnels) $\left.{ }^{1}\right)$. Désignons par $U_{\Xi}$ l'ensemble des $x$ tels que le type d'ordre de l'ensemble $M_{x}$ est $\xi: \bar{M}_{x}=\xi$. Done, $U$ désignant le complémentaire de $A$, on a $U=\sum_{i<0} U_{\xi}$; c'est la décomposition de $U$ en "constituantes".

De plus, comme l'a remarqué M. Novikoff $\left.{ }^{2}\right), \delta$ étant un nombre $<\Omega$ donné en avance; on peut s'arranger de facon que les

1) Cf. par exemple ma Topologie $I$, p. 255.

2) Fund. Matl. 25, p. 462 . indices des ensembles $U_{\xi}$ non vides soient de la forme $\xi=\xi^{*}+\delta$. Il suffit à ce but de définir dans l'intervalle $(1,2)$ un ensemble $\Delta$ de nombres rationnels dont le type d'ordre soit $\delta$ et d'admettre que, poür $r \in \Delta$, l'ẹsemble $W_{r}$ coïncide avec l'espace tout entier.

Passons à présent à la démonștration du théorème. $U^{1}, U^{2}, \ldots$ étant une suite infinie d'ensembles $C A$, posons $U^{n}=\sum_{\xi<\Omega} U_{\xi}^{n}$, où $U_{\xi}^{n}$ désignent les constituantes de $U^{n}$. En vertu de la remarque faite tout-à-l'heure, admettons que les indices des constituantes non vides soient de la forme $\xi+\omega^{n}$. Par conséquent,

(6) pour $n \neq m$ on a, soit $U_{\xi}^{n}=0$, soit $U_{\xi}^{m}=0$, quel que soit $\xi$.

La formule (4) du $N^{0} 1$ étant ainsi vérifiée, il s'agit de démontrer que l'ensemble $V^{\prime \prime}$ défini par l'égalité (5) est de classe $C A$. Or, l'indice $\beta$ n'admettant que les valeurs finies, tout se réduit à démontrer que l'ensemble $D_{\alpha \beta}=\sum_{S}\left(S_{\xi}^{\prime c}-S_{\xi}^{\beta}\right)$ est de classe $C A$.

$D_{\alpha \beta}$ est l'ensemble des $x$ pour lesquels il existe un $\xi<\Omega$ tel que $\bar{M}_{x}^{\prime i} \leqslant \xi$ et que $\bar{M}_{x}^{\beta}$ non $\leqslant \xi$. C'est pąr conséquent l'ensemble des $x$ tels que $1^{0}$ l'ensemble $M_{x}^{u}$ est bien ordonné et que $2^{0} 1^{\prime}$ 'ensemble $M_{x}^{3}$ n'est pas semblable à un sous-ensemble de $M_{x}^{c i}$ (considéré comme ensemble ordonné). Or l'ensemble des $x$ satisfaisaht à la condition $1^{0}$ étant identique à $U^{\prime \prime}$ et celui des $x$ qui satisfont à la condition $2^{0}$ étant un $C A$, selon un théorème de $\mathrm{M}$. $\mathrm{L}$ u $\sin ^{1}$ ), l'ensemble $D_{a f f}$, comme produit de ces deux ensembles, est aussi un $C A$.

\section{Application aux ensembles $P C A$.}

La famille des ensembles PCA satisfait au théorème de réduction généralisé.

Démonstration. ${ }^{2}$ ) Nous commençons la démonstration par quelques remarques qui se rattachent à la démonstration précédente. Soit $A$ un ensemble analytique dans, un espace complet sé-

1) Pour une simple démonstration de ce théorème très remarquable, voir par ex. Topologie I, p. 258.

$\left.{ }^{2}\right)$ Nous suivons ici l'idée générale de la démonstration de M. Novik off (l. cit.), en la simplifiant légèrement. 
parable $Y$ et soient $W_{r}, M_{y}$ et $U_{\Xi}$ les ensembles considérés dans le $\mathbb{N}^{0}$ précédent. $Z$ étant un espace complet séparable donné, les ensembles $W_{r} \times Z^{1}$ ) constituent évidemment un crible pour l'ensemble $A \times Z$ et cette multiplication n'altère pais le type d'ordre de l'ensemble $M_{y}$, c. à d. que $\bar{M}_{y z}=\bar{M}_{y}$. Par conséquent, les ensembles $\underset{\| z}{\mathrm{E}}\left(y \epsilon U_{\xi}\right)=U_{\xi} \times Z$ sont des constituantes du complémentaire de l'ensemble analytique $\mathrm{E}(y \in A)=A \times Z$.

Cela étant, soit $P^{1}, \stackrel{{ }^{2}}{P^{2}}, \ldots$ une suite infinie d'ensembles $P C A$. Il existe, par conséquent, une suite $B^{1}, B^{2}, \ldots$ d'ensembles $C A$ tels que $P^{n}$ soit une projection de $B^{n}$, c. à d. que $P^{n}=\sum_{y} \underset{x}{\mathbb{X}}\left(x y \in B^{n}\right)$. Soit $B^{n}=\sum_{\Omega} U_{\xi}^{n}$ le développement de $B^{n}$ en constituantes. Nous pouvons supposer que la condition (6) èst réalisée.

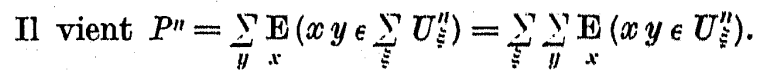

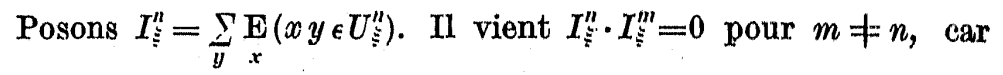
autrement il existerait un $y$ tel que $x y \in U_{\xi}^{\prime \prime}$, on aurait done $U_{\xi}^{\prime \prime} \neq 0$ et, de même $U_{⿱ 亠 䒑}^{m} \neq 0,-$ contrairement à $(6)$.

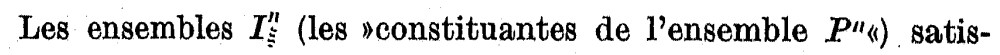
font done à la condition (4).

En vertu de (5) tout revient à démontrer que l'ensemble $J_{\alpha \beta}=\sum_{\Omega}\left[\left(I_{1}^{\alpha}+\ldots+I_{\breve{\xi}}^{\alpha}\right)-\left(I_{1}^{\beta}+\ldots+I_{\xi}^{\beta}\right)\right]$ est un $P O A$.

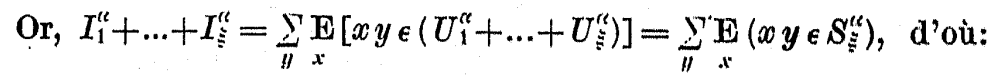

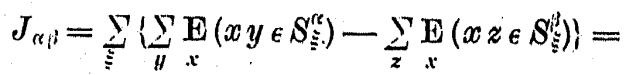

$$
\begin{aligned}
& =\sum_{i} \sum_{y} \prod_{z}\left\{\mathrm{E}\left(x y \in S_{\xi}^{(k)}\right)-\sum_{x}^{\mathrm{E}}\left(x z \in S_{\xi}^{\mathrm{B}}\right)\right\}=
\end{aligned}
$$

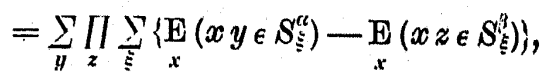

car on peut remplacer toujours $\sum_{\xi} \sum_{y}$ par $\sum_{\bar{y}} \sum_{\bar{\xi}}$ et puis on remplace

1) $Y \times Z$ désigne le produit cartésien de $Y$ et $Z$, c. à d. l'ensemble des couples $(y, z)$ où $y \in Y$ et $z \in Z$. $\sum_{\xi} \prod_{z} \operatorname{par} \prod_{z} \sum_{\xi}$ en vertu de la formule générale suivante

$$
\quad \sum_{\xi} \prod_{z} L_{\xi} \cdot K_{\xi, z}=\prod_{z} \sum_{\xi} L_{\xi} \cdot K_{\xi, z}
$$

qui est valable lorsque pour chaque $z$ on a $K_{1, z} \supset K_{2, z} \supset \ldots{ }^{1}$ ).

Posons, pour abréger

$$
\begin{aligned}
\dot{U}_{\xi}=\underset{x y z}{\mathrm{E}} x y \in U_{\xi}^{\prime \prime} & \ddot{U}_{\xi}=\underset{x y z}{\mathrm{E}} x z \in U_{\xi}^{u} \\
\dot{S}_{\xi}=\dot{U}_{1}+\ldots+\dot{U}_{\xi} & \ddot{S}_{\xi}=\ddot{U}_{1}+\ldots+\ddot{U}_{\xi} .
\end{aligned}
$$

D'après la remarque faite au début, les ensembles $\dot{U}_{\xi}$ (ainsi que $\ddot{U}_{\xi \xi}$ ) présentent un système de constituantes du complémentaire d'un ensemble analytique. Donc, comme nous l'avons démontré dans le $\mathrm{N}^{0} 2$, l'ensemble $\sum_{\xi}\left(\dot{S}_{\xi}-\ddot{S}_{\xi}\right)$ est un ensemble $C A$.

D'autre part, $\underset{x}{\mathrm{E}} x y \in \dot{S}_{\xi}^{\alpha}=\underset{x}{\mathrm{E}} x y z \in \dot{S}_{\xi}$, d'où

$$
\begin{aligned}
& J_{\alpha \beta}=\sum_{y} \prod_{z} \sum_{\grave{\xi}} \underset{x}{\mathrm{E}}\left[x y z \epsilon\left(\dot{S}_{\grave{\xi}}-\ddot{S}_{\xi}\right)\right]= \\
& =\sum_{y} \prod_{z} \underset{x}{\mathrm{E}}\left\{x y z \in \underset{\xi}{\sum_{\xi}}\left(\dot{S}_{\xi}-\ddot{S}_{\xi}\right)\right\} .
\end{aligned}
$$

Il en résulte ${ }^{2}$ ) que l'ensemble $J_{\kappa \beta}$ est de classe $P C P C C A$, c. à d. de classe $P C A^{3}$ ).

1) Il s'agit de prouver que, si $p$ appartient au membre droit, il appartient au membre gauche. Or, il existe par hypothèse pour chaque $z$ un nombre $\xi z$ tel que $p \in L_{\xi_{z}} \cdot K_{\xi_{z}, z}$; soit $\alpha$ le plus petit parmi ces $\xi z$ (pour $p$ fixe). Donc $p \in L_{1 t}$ ot, comme $\alpha \leqslant \xi z$ quel que soit $z$, il vient $p \in K_{\xi_{z}, z} \subset K_{a, x}$. D'où la conclusion demandée.

Dans le cas considéré on pose $L_{\S}=\underset{x}{\mathrm{E}}\left(x y \in S_{\xi}^{(\alpha)}\right)$ et $K_{\S, z}=\underset{x}{\mathrm{E}}\left(x z\right.$ non- $\left.\epsilon S_{\xi}^{\natural}\right)$.

2) D'une façon générale, si un ensemble $T$ contenu dans le produit $X \times Y$ est de classe projective $L$, l'ensemble $\sum_{y} \underset{x}{\mathrm{E}}(x y \in T)$ est de classe $P L$ et l'ensemble $\prod_{y} \underset{x}{\mathrm{E}}(x y \in T)$ est de clasise $O P O L$. Voir par ex. ma Topologie I, § 34, VIII.

$$
\text { On a ici } T=\sum_{\xi}\left(\dot{S}_{\xi}-\ddot{S}_{\xi}\right) \subset X \times Y \times Z \text {. }
$$

a) Une démonstration du fait que $J_{\text {ç̣ }}$ est un $P C A$, basée sur une idée différente (celle de ,suite projective d'ensembles"), paraitra prochaînement. 


\section{Théorèmes de séparation.}

Admettons, à présent, que la famille $\boldsymbol{B}$ satisfait au théorème de réduction généralisé et qu'en outre elle est additive au sens dénombrable (c. à d. qu'elle contient la somme de toute série composée de ses éléments). Soit $\boldsymbol{A}$ la famille des complémentaires des ensembles appartenant à $\boldsymbol{B}$; des familles $\boldsymbol{A}$ forment, en particulier: $1^{0}$ les ensembles boreliens de classe $\beta>0$ multiplicative, $2^{0}$ les ensembles analytiques, $3^{0}$ les ensembles $C P O A$.

Le théorème de réduction généralisé implique les énoncés suivants.

\section{Premier théorème de séparation (généralisé) ${ }^{1}$ ).}

Etant donnée une suite d'ensembles $A_{1}, A_{2}, \ldots$ appartenant d ${ }^{A}$ et tels que $\prod_{n=1}^{\infty} A_{n}=0$, il existe une suite d'ensembles $B_{n}$ qui, de méme que leurs complémentaires, appartiennent $\dot{a} \boldsymbol{A}$ et qui satisfont aux conditions

$$
A_{n} \subset B_{n} \text { et } \prod_{n=1}^{\infty} B_{n}=0 .
$$

Appliquons le théorème de réduction généralisé à la famille $\boldsymbol{B}$ en posant $U^{n}=X-A_{n}$ et $B_{n}=X-V^{n}$ ( $X$ désignant l'espace). Par hypothèse $X=\sum_{n} U^{n}=\sum V^{n}$. Les ensembles $V^{n}$ étant disjoints, il vient $X-\dot{V}^{n}=\sum_{i \neq n} V^{i}$, d'où on conclut que $X-V^{n}$ appartient à $\boldsymbol{B}$, donc que $V_{n}$ appartient à $A$. En outre, $\prod_{n} B_{n}=X-\sum_{n} V^{n}=0$.

2. Deuxième théorème de séparation (généralisé) ${ }^{2}$ ).

Etant donnée une suite d'ensembles $A_{1}, A_{2}, \ldots$ appartenaint à $A$, il existe une suite d'ensembles $C_{1}, C_{2}, \ldots$ dont les complémentaires appartiennent $\dot{a} \boldsymbol{A}$ et qui satisfont aux conditions

$$
A_{n}-\prod_{m=1}^{\infty} A_{m} \subset_{-} C_{n} \text { et } \prod_{n=1}^{\infty} C_{n}=0
$$

1) Pour les ensembles boreliens d'une classe multiplicative, démontré par M. Sierpiński, Fund. Math. 23, p. 295; pour les ensembles analytiques par M. Novikoff, C. R. Acad. Sc. URSS, t. II (1934), p. 145; pour les ensembles $C P C A$ par le même auteur dans Fund. Math. 25, p. 465 .

2) Pour le cas des ensembles OPOA voir Novikoff, l, c. p. 466.
Posons, en effet, $U^{n}=X-A_{n}$ et $C_{n}=\sum_{i \neq n} V^{i} . \mathrm{Il}$ vient

$$
\begin{gathered}
A_{n}-\prod_{m} A_{m}=\sum_{m} U^{m}-U^{n}=\sum_{m} V^{m}-U^{n}=\left(C_{n}+V^{n}\right)-U^{n}= \\
=\left(O_{n}-U^{n}\right)+\left(V^{n}-U^{n}\right)=C_{n}-U^{n} \subset C_{n} .
\end{gathered}
$$

En outre, comme $C_{n} \cdot \nabla^{n}=0$, on a $\prod_{n} C_{n} \cdot \sum_{n} V^{n}=0$. Rapprochée de l'inclusion $C_{n} \subset \sum_{m} V^{m}$, qui implique $\prod_{n}^{n} C_{n} \subset_{n}^{n} \sum_{n} V^{n}$, cette égalité donne $\prod_{n} C_{n}=0$.

Ajoutons, à présent, aux hypothèses faites sur $\boldsymbol{A}$ celle qu'elle soit additive au sens dénombrable. Telles sont en particulier les familles des ensembles analytiques et des ensembles $C P C A$.

On a le théorème de séparation suivant

3. $\left.{ }^{1}\right)$ Etant donnée une suite d'ensembles $A_{1}^{*}, A_{2}^{*}, \ldots$ appartenant $\grave{a} \boldsymbol{A}$ et tels que $\operatorname{Lim} \sup A_{n}^{*}=0$, il existe une suite d'ensembles $D_{n} q u i$, de même que leurs complémentaires, appartiennent d̀ $\boldsymbol{A}$ et qui satisfont aux conditions

$$
A_{n}^{*} \subset D_{n} \quad \text { et } \operatorname{Lim} \sup D_{n}=0 .
$$

C'est une conséquence facile de l'énoncé 1 .

Posons, en effet, $A_{n}=A_{n}^{*}+A_{n+1}^{*}+\ldots$ On a par hypothèse II $A_{n}=0$ ei le premier théorème de séparation est applicable. Posons

$$
D_{1}=B_{1} \text { et, en général, } D_{n}=D_{n-1} \cdot B_{n} \text {. }
$$

Il vient $A_{n}^{*} \subset A_{n} \subset B_{n}$ et $A_{n} \subset A_{n-1}$; en admettant (par induction) que $A_{n-1} \subset D_{n-1}$, on en tire que $A_{n}^{*} \subset B_{n} \cdot D_{n-1}=D_{n}$.

En outre, comme $D_{n} \subset D_{n-1}$, on a

$$
\operatorname{Lim} \sup D_{n}=\prod_{n=1}^{\infty} \sum_{i=0}^{\infty} D_{n+i}=\prod_{n=1}^{\infty} D_{n} \subset \prod_{n=1}^{\infty} B_{n}=0 .
$$

1) Pour les ensembles analytiques démontré par M. Liapou noff, C. R. Acad. Se. URSS, t. II (1934), p. 276. 